\title{
Effect of a Dual-Strain Probiotic on Necrotizing Enterocolitis in Neonates with Ductal-Dependent Congenital Heart Disease: A Retrospective Cohort Study
}

\author{
Liz Kocjancic $^{a}$ Christoph Bührer $^{\mathrm{a}}$ Felix Berger ${ }^{\mathrm{b}, \mathrm{c}}$ Vinzenz Boos ${ }^{\mathrm{a}} \mathrm{b}$ \\ ${ }^{a}$ Department of Neonatology, Charité - Universitätsmedizin Berlin, Berlin, Germany; ${ }^{b}$ Department of Congenital \\ Heart Disease/Pediatric Cardiology, German Heart Center Berlin, Berlin, Germany; ' German Center for \\ Cardiovascular Research (DZHK), Congenital Heart Diseases, Berlin, Germany
}

\author{
Keywords \\ Congenital heart disease $\cdot$ Necrotizing enterocolitis . \\ Probiotics · Prostaglandin E1
}

\begin{abstract}
Background: Newborns with ductal-dependent congenital heart disease (CHD) are at increased risk for developing necrotizing enterocolitis (NEC). Objectives: To investigate whether the use of dual-strain probiotics is beneficial for prevention of NEC in CHD patients, as demonstrated for premature infants. Study Design: Single-center retrospective cohort study of newborns with ductal-dependent CHD before and after implementation of oral dual-strain probiotics containing Bifidobacterium infantis and Lactobacillus acidophilus, on each day of exposure to prostaglandin E1 (PGE1). Results: Birth weight, gestational age, and distribution of heart defects were similar in both cohorts. NEC occurred in 6 of 247 (2.4\%) patients without probiotics, and in 3 of 242 (1.2\%) patients who received probiotics ( $p=0.504)$. NEC-related mortality ( 0.4 vs. $0.4 \%, p=1.000)$ and overall mortality (11.0 vs. $8.7 \%, p=0.448$ ) were likewise not different. PGE1 exposure was 1,788 and 2,455 days, respectively. In subgroup analysis of 152 infants with aortic arch malformations, such as coarc-
\end{abstract}

tation of the aorta and interrupted aortic arch, we observed a significant reduction of NEC frequency ( 5.6 vs. $0.0 \%, p=$ 0.048). Conclusions: This is the first study to investigate the effect of a dual-strain probiotic on NEC in CHD patients. Infants with aortic arch malformations appear to benefit from dual-strain probiotics. Due to the scarcity of concurrence of ductal-dependent CHD and NEC, a clinical trial on probiotics to decrease risk of NEC in infants with ductal-dependent $\mathrm{CHD}$ would require several thousand infants.

(c) 2020 S. Karger AG, Basel

\section{Introduction}

Necrotizing enterocolitis (NEC) is a devastating gastrointestinal disease and predominantly affecting premature infants [1]. Presence of a congenital heart disease (CHD), particularly a complex or ductal-dependent $\mathrm{CHD}$, is a major risk factor for the development of NEC in near-term and full-term neonates [2-4]. The incidence of NEC in term infants with CHD has been reported to be $3-11 \%$, which is substantially higher than rates reported for the entire population of late preterm and term newborns $[2,5,6]$. karger@karger.com

(c) 2020 S. Karger AG, Basel

www.karger.com/neo

Karger ${ }^{\prime}=$
Vinzenz Boos

Department of Congenital Heart Disease/Pediatric Cardiology

German Heart Center Berlin

Augustenburger Platz 1, DE-13353 Berlin (Germany)

boos@dhzb.de 
Fig. 1. Flow diagram of patient enrollment.

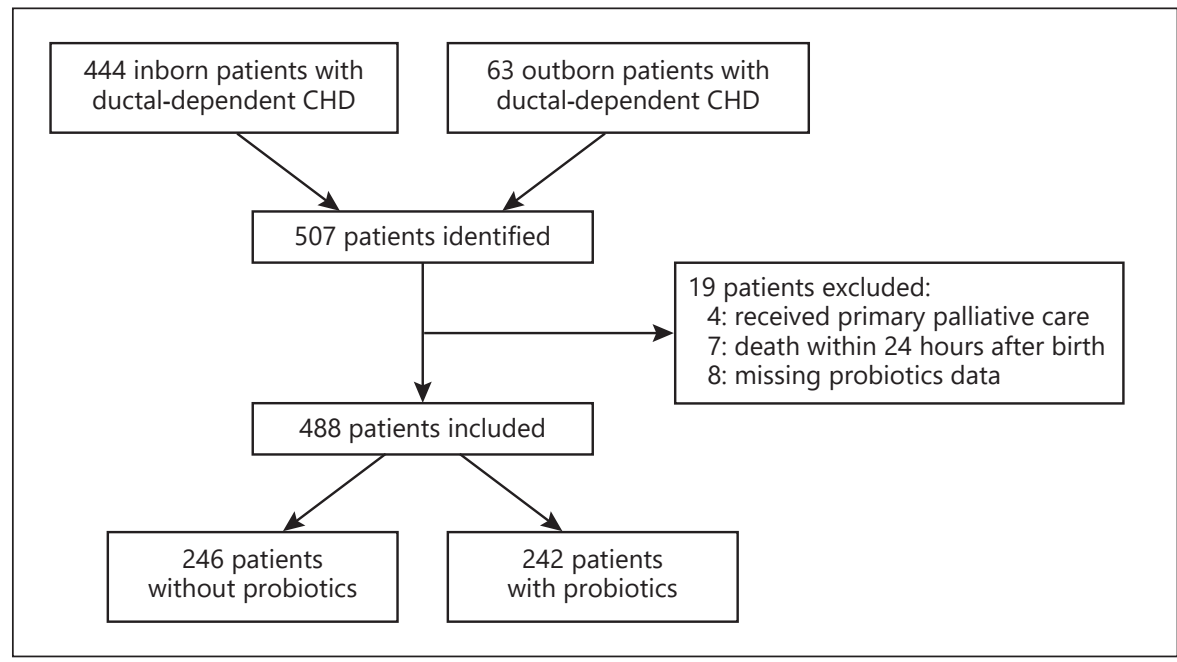
$\mathrm{CHD}$, congenital heart disease.

The pathogenesis of NEC is multifactorial and incompletely understood, but genetic predisposition, alterations in gut flora, inadequate intestinal barrier function, regulation of the gastrointestinal and systemic inflammatory response, and hypoxia/ischemia might contribute to intestinal necrosis $[7,8]$. Enteral supplementation of probiotics has the potential to change the intestinal microbiota, leads to a reduction in the frequency of NEC in very low birth weight (VLBW) premature infants, and has become one of the most studied interventions in neonatal medicine in recent years $[9,10]$.

In contrast to prematures, the underlying cardiac disease can lead to abnormal vascular flow in mesenteric vessels and intestinal hypoxia-ischemia in CHD patients, with secondary inflammation [11]. The role of microbial bowel colonization in the pathogenesis of NEC in CHD patients is unknown, but intestinal hypoperfusion and hypoxia, low cardiac output, antibiotic therapies, and delayed enteral feeding due to respiratory or cardiovascular instability might, alone or in combination, alter the intestinal microbiota in these patients $[12,13]$.

No study has yet investigated whether the supplementation of probiotics influences the risk for NEC in neonates with CHD $[12,14]$. Following the emergence of strong evidence that probiotics reduce rates of NEC and mortality in premature infants, the most widely studied probiotics consisting of Bifidobacterium infantis and Lactobacillus acidophilus were routinely administered to all newborns considered at increased risk of NEC [15]. VLBW premature infants and infants with CHD requiring continuous infusion of prostaglandin E1 (PGE1) were equally considered to be at risk of NEC, and the shift in institutional policy was indeed associated with a NEC reduction in VLBW infants $[2,16]$. In this retrospective cohort study, we investigate the potential influence of a dual-strain probiotic containing Bifidobacterium infantis and Lactobacillus acidophilus on newborns with ductaldependent CHD.

\section{Materials and Methods}

\section{Inclusion Criteria}

A retrospective analysis was performed on all neonates born between January 2005 and December 2014 with ductal-dependent CHD, who received PGE1 to maintain ductal patency. Exclusion criteria were death within $24 \mathrm{~h}$ after birth, primary palliative care, and missing data on the administration of probiotics.

\section{Intervention}

The routine use of probiotics during administration of PGE1 in children with ductal-dependent CHD was gradually implemented during 2009. Patients received $1 / 2$ capsule of Infloran ${ }^{\circledR}$ (Laboratorio Farmaceutico, Mede, Italy) containing Bifidobacterium infantis and Lactobacillus acidophilus twice daily (total daily dose $1 \times 10^{9}$ colony-forming units of each organism) on each day of PGE1 exposure. Two subgroups were formed for comparison: the control group of patients without any supplementation of probiotics and the group of infants receiving enteral probiotics during PGE1 administration. Enteral feeding was routinely started within 24 hours after birth with maternal breast milk, if available, or formula.

\section{Study Measures}

All data were retrieved from medical files. Extracted data for each patient include demographic details, type of ductal-dependent CHD, duration of PGE1 administration, data on the perinatal and perioperative clinical course and management, NEC, and mortalities, until hospital discharge. 
Table 1. Demographic data and clinical course

\begin{tabular}{|c|c|c|c|}
\hline Variable & No probiotics $(n=246)$ & Probiotics $(n=242)$ & $p$ value \\
\hline \multicolumn{4}{|l|}{ Demographic data ${ }^{\mathrm{a}}$} \\
\hline Sex, male & $154(62.6)$ & $133(55.0)$ & 0.086 \\
\hline Gestational age, weeks & $38.7(25.4-42.0)$ & $38.6(28.1-41.4)$ & 0.945 \\
\hline Prematurity (<37 weeks' gestational age) & $46(18.8)$ & $36(14.9)$ & 0.250 \\
\hline Birth weight, $g$ & $3,205(640-4,800)$ & $3,110(706-4,650)$ & 0.437 \\
\hline Very low birth weight $(<1,500 \mathrm{~g})$ & $8(3.3)$ & $7(2.9)$ & 0.812 \\
\hline Multiple gestation & $17(6.9)$ & $16(6.6)$ & 0.895 \\
\hline \multicolumn{4}{|l|}{ Data on delivery ${ }^{\mathrm{a}}$} \\
\hline Inborn delivery & $207(83.7)$ & $221(91.3)$ & 0.011 \\
\hline Caesarean section & $86(35.0)$ & $110(45.5)$ & 0.021 \\
\hline Apgar score at $5 \min ^{\mathrm{b}}$ & $9(2-10)$ & $9(1-10)$ & 0.010 \\
\hline \multicolumn{4}{|l|}{ Postnatal clinical course $e^{\mathrm{a}, \mathrm{c}}$} \\
\hline Early-onset infection, suspected ${ }^{\mathrm{d}}$ & $42(17.8)$ & $32(13.2)$ & 0.167 \\
\hline Late-onset infection, suspected ${ }^{\mathrm{d}}$ & $13(5.5)$ & $17(7.0)$ & 0.487 \\
\hline Postnatal antibiotic therapy & $131(53.3)$ & $107(44.4)$ & 0.051 \\
\hline Duration of antibiotic therapy, days & $4(1-91)$ & $3(1-37)$ & 0.078 \\
\hline Hemoglobin at birth, g/L & $172(88-247)$ & $172(56-236)$ & 0.977 \\
\hline Red blood cell transfusion & $34(14.0)$ & $31(12.8)$ & 0.702 \\
\hline Platelet transfusion & $6(2.4)$ & $7(2.9)$ & 0.761 \\
\hline Mechanical ventilation & $110(45.1)$ & $77(32.0)$ & 0.003 \\
\hline Administration of surfactant & $14(5.7)$ & $4(1.7)$ & 0.028 \\
\hline \multicolumn{4}{|l|}{ Cardiac management ${ }^{\mathrm{a}}$} \\
\hline Cardiac catheterization including BAS, pre & $120(49.2)$ & $123(50.8)$ & 0.717 \\
\hline PGE1 infusion & $246(100.0)$ & $242(100.0)$ & $\mathrm{N} / \mathrm{A}$ \\
\hline PGE1, duration of administration, days & $6(1-99)$ & $8(1-60)$ & $<0.001$ \\
\hline PGE1, total duration of exposure, infant days & 1,788 & 2,455 & \\
\hline
\end{tabular}

Data are presented as number of patients (\%) or median (range). ${ }^{\text {a }}$ The following information (No. of infants) was missing: gestational age $=1$, prematurity $=1$, birth weight $=1$, very low birth weight $=1$, Caesarean delivery $=2$, Apgar score $=3$, early-onset infection $=10$, late-onset infection $=9$, duration of antibiotic therapy $=16$, hemoglobin at birth $=43$, red blood cell transfusion $=3$, platelet transfusion $=1$, mechanical ventilation $=2$, administration of surfactant $=1$, cardiac catheterization $=2$, PGE1 duration $=4$ in the no probiotics group, and Apgar score $=1$, postnatal antibiotic therapy $=1$, duration of antibiotic therapy $=2$, hemoglobin at birth $=17$, mechanical ventilation $=1$, PGE 1 duration $=1$ in the probiotics group. ${ }^{\mathrm{b}} 25$ th and 75 th centiles are 8 and 9 for the control group, and 8 and 10 for the probiotics group, respectively. ${ }^{\mathrm{c}}$ Before cardiac surgery or discharge. ${ }^{\mathrm{d}}$ Based on clinical signs and laboratory tests (interleukin-6, C-reactive protein, and hematological parameters).

\section{Outcomes}

Primary outcome of our study was diagnosis of Modified Bell Stage IIa or higher NEC, either before or following cardiac surgery [17]. Secondary outcomes were NEC-related mortality, defined as mortality attributed to worsening of NEC, and overall mortality before discharge from hospital. Results of blood cultures were obtained for safety analysis.

\section{Statistics}

The data were analyzed using IBM SPSS Statistics, version 24.0 (IBM Inc., Armonk, NY, USA). Descriptive data for continuous variables are presented as median and range, categorical variables as relative frequencies. Differences for continuous variables between the two groups were analyzed using Mann-Whitney U test. Chi-square and two-tailed Fisher's exact test were used for com-

Probiotics for NEC Prevention in

Neonates with Congenital Heart Disease parison of dichotomous variables. Sample size calculation was performed by the Kelsey method using OpenEpi, version 3.01 (Open Source Epidemiologic Statistics for Public Health, Atlanta, GA, USA). A $p$ value $<0.05$ was considered statistically significant.

\section{Results}

Inclusion criteria were met by 507 patients. Four patients with primary palliative care, 7 patients who died within 24 hours after birth, and 8 patients with missing data on administration of probiotics were excluded. The study cohort of 488 patients was divided into two sub- 
Table 2. Types of congenital heart malformations in the study cohort

\begin{tabular}{lccc}
\hline Cardiac lesion & $\begin{array}{l}\text { No probiotics } \\
(n=246)\end{array}$ & $\begin{array}{l}\text { Probiotics } \\
(n=242)\end{array}$ & $\begin{array}{l}p \\
\text { value }\end{array}$ \\
\hline Aortic atresia + coarctation of the aorta & $0(0.0)$ & $1(0.4)$ & 0.496 \\
Aortic atresia + interrupted aortic arch & $0(0.0)$ & $1(0.4)$ & 0.496 \\
Aortic stenosis & $7(2.8)$ & $3(1.2)$ & 0.339 \\
Aortic stenosis + coarctation of the aorta & $4(1.6)$ & $3(1.2)$ & 1.000 \\
Coarctation of the aorta & $60(24.4)$ & $57(23.6)$ & 0.829 \\
Dextro-transposition of the great arteries & $76(30.9)$ & $74(30.6)$ & 0.940 \\
Dextro-transposition of the great arteries + coarctation of the aorta & $4(1.6)$ & $11(4.5)$ & 0.074 \\
Double outlet right ventricle & $3(1.2)$ & $2(0.8)$ & 1.000 \\
Ebstein's anomaly & $4(1.6)$ & $2(0.8)$ & 0.687 \\
Hypoplastic left heart syndrome & $30(12.2)$ & $29(12.0)$ & 0.943 \\
Interrupted aortic arch & $3(1.2)$ & $7(2.9)$ & 0.219 \\
Pulmonary atresia & $19(7.7)$ & $26(10.7)$ & 0.249 \\
Pulmonary stenosis & $7(2.8)$ & $6(2.5)$ & 0.802 \\
Shone's complex & $2(0.8)$ & $1(0.4)$ & 1.000 \\
Tricuspid atresia & $3(1.2)$ & $3(1.2)$ & 1.000 \\
Total anomalous pulmonary venous return & $5(2.0)$ & $1(0.4)$ & 0.216 \\
Tetralogy of Fallot & $7(2.8)$ & $7(2.9)$ & 0.975 \\
Unbalanced atrioventricular septal defect & $2(0.8)$ & $2(0.8)$ & 1.000 \\
Others ${ }^{a}$ & $10(4.1)$ & $6(2.5)$ & 0.325 \\
\hline
\end{tabular}

Data are presented as number of patients (\%). ${ }^{\mathrm{a}}$ Multiple cardiac anomalies including other ductal-dependent single ventricle anomalies.

groups: 246 patients (50.4\%) who did not receive any enteral probiotics formed the control group, and $242 \mathrm{pa}-$ tients $(49.6 \%)$ with administration of dual-strain probiotics formed the intervention group (Fig. 1).

\section{Patients' Characteristics}

Demographic characteristics did not differ between the two groups. The rate of inborn births was increased, and there were more Caesarean deliveries in the intervention group (Table 1). The rate of suspected early- and late-onset infections did not differ between the two groups, but we observed an insignificant trend towards less frequent and shorter postnatal antibiotic courses in the intervention group. Two infants who developed postoperative NEC in the control group had received transfusions of red blood cells after cardiac surgery, 1 or 2 days, respectively, before onset of NEC. No other infant was diagnosed with NEC within $48 \mathrm{~h}$ of a blood transfusion. Patients who received probiotics were significantly longer exposed to PGE1 (median 6 vs. 8 days, $p<0.001$ ), and the amount of total days of PGE1 exposure was $37 \%$ higher in the probiotic group compared to the control group, with 2,455 vs. 1,788 days (Table $1)$. The frequencies of various types of ductal-dependent CHD were similarly distributed between the two groups in our study cohort (Table 2). Most common lesions were dextro-transposition of the great arteries and coarctation of the aorta, both also with additional variations.

\section{Outcomes}

There was no significant difference in the frequency of NEC ( 2.4 vs. $1.2 \%, p=0.504)$, NEC-related mortality ( 0.4 vs. $0.4 \%, p=1.000$ ), and overall mortality ( 11.0 vs. $8.7 \%$, $p=0.448)$ between the two study groups. NEC was diagnosed on median day 10 of life (5-35 days). One patient of the control group was treated conservatively, whereas 8 out of 9 infants with NEC required abdominal surgery. The diagnosis of NEC was additionally confirmed by characteristic findings, as seen during laparotomy or in histological examinations in 7 of these. In one infant who received probiotics, histological examination revealed cecal perforation with local inflammation, and spontaneous intestinal perforation could also be considered for differential diagnosis in this patient. We found no significant difference in outcomes in the subgroups of VLBW and non-VLBW infants (Table 3). Subgroup analysis of infants stratified by types of CHD revealed a reduction of NEC incidence in patients with aortic arch malformations leading to decreased perfusion of the aorta (5.6 vs. 
Table 3. Effect of probiotics on NEC and mortality stratified by birth weight

\begin{tabular}{|c|c|c|c|}
\hline Variable & No probiotics & Probiotics & $p$ value \\
\hline All infants & $246(100.0)$ & $242(100.0)$ & \\
\hline NEC, overall & $6(2.4)$ & $3(1.2)$ & 0.504 \\
\hline NEC, after cardiac surgery & $3(1.2)$ & $0(0.0)$ & 0.249 \\
\hline NEC-related mortality ${ }^{\mathrm{a}}$ & $1(0.4)$ & $1(0.4)$ & 1.000 \\
\hline Mortality, overall & $27(11.0)$ & $21(8.7)$ & 0.448 \\
\hline Birth weight $<1,500 \mathrm{~g}^{\mathrm{b}}$ & $8(100.0)$ & $7(100.0)$ & \\
\hline NEC, overall ${ }^{c}$ & $2(25.0)$ & $1(14.2)$ & 1.000 \\
\hline NEC, after cardiac surgery & $0(0.0)$ & $0(0.0)$ & 1.000 \\
\hline NEC-related mortality & $1(12.5)$ & $1(14.2)$ & 1.000 \\
\hline Mortality, overall & $6(75.0)$ & $2(28.6)$ & 0.132 \\
\hline Birth weight $\geq 1,500 \mathrm{~g}^{\mathrm{b}}$ & $237(100.0)$ & $235(100.0)$ & \\
\hline NEC, overall ${ }^{c}$ & $4(1.7)$ & $2(0.9)$ & 0.686 \\
\hline NEC, after cardiac surgery & $3(1.3)$ & $0(0.0)$ & 0.248 \\
\hline NEC-related mortality & $0(0.0)$ & $0(0.0)$ & $\mathrm{N} / \mathrm{A}$ \\
\hline Mortality, overall & $21(8.9)$ & $19(8.1)$ & 0.869 \\
\hline
\end{tabular}

Data are presented as number of patients (\%). ${ }^{a}$ Patient with preoperative onset of NEC. ${ }^{b}$ Information on birth weight was missing for one patient in the no probiotics group, who was $35^{1} / 7$ weeks of gestational age at birth and did not develop NEC. ${ }^{c}$ Difference between frequency of NEC in VLBW infants and infants with birth weight $\geq 1,500$ g: $p=0.013$ (no probiotics group) and $p=0.085$ (probiotics group), respectively.

$0.0 \%, p=0.048)$, such as coarctation of the aorta and interrupted aortic arch (Table 4).

\section{Probiotic Bacteremia}

A total of 86 patients (35.5\%) of the intervention group had blood cultures, all being negative for Bifidobacterium or Lactobacillus bacteremia. Organisms identified were Acinetobacter or Staphylococcus in 2 blood cultures in the control group, and Escherichia coli, Staphylococcus, or Streptococcus in 6 blood cultures in the intervention group.

\section{Sample Size Calculation}

A randomized controlled trial with the aim to demonstrate a significant reduction of NEC incidence from 2.4 to $1.2 \%$ by a single intervention, such as administration of probiotics, would need to include at least 3,786 patients $(1,893$ in each group, allocation ratio $1: 1)$, for a two-sided type I error of $5 \%$ and a power of $80 \%$.

\section{Discussion}

Our results indicate that oral administration of a dualstrain probiotic might be beneficial to reduce the rate of NEC in infants with aortic arch malformations. The low frequencies of overall and postoperative NEC in our center are rather in the lower range of the various NEC frequencies reported in previous studies, making analyses whether the administration of probiotics has an influence on the frequency of NEC in CHD patients challenging $[2,5,6]$.

Differences between both groups, notably more Caesarean deliveries and a trend towards less frequent antibiotic use in the intervention group, might impact our results. Both factors have been shown to influence the composition of the intestinal microbiota by reducing the amount of Bifidobacteria and Bacteroides fragilis group in fecal samples in infants at 1 month of age [18]. However, the impact of mode of delivery and antibiotic therapy on NEC incidence is still critically discussed [19]. Furthermore, we could not investigate whether the infants were fed with breast milk or formula. Breast milk has been shown to have a beneficial effect in reducing the incidence of NEC in premature infants; however, in contrast, no difference in NEC incidence was found between infants with ductal-dependent CHD fed with breast milk or formula $[19,20]$.

Growing evidence leads to the assumption that NEC in CHD patients is a clinically distinct entity from NEC in premature infants, distinguished by different demographics, pathogenic mechanisms, and outcomes $[6,11$, 12]. Previous studies show contradictory results regarding the age at onset of NEC in CHD patients, especially in 
Table 4. Effect of probiotics on NEC and mortality stratified by type of CHD

\begin{tabular}{lccc}
\hline Variable & No probiotics & Probiotics & $p$ value \\
\hline CoA and IAA, all $(n=152)^{\mathrm{a}}$ & $72(100.0)$ & $80(100.0)$ & \\
NEC, overall & $4(5.6)$ & $0(0.0)$ & 0.048 \\
NEC, after cardiac surgery & $2(2.8)$ & $0(0.0)$ & 0.223 \\
NEC-related mortality & $1(1.4)$ & $0(0.0)$ & 0.474 \\
Mortality, overall & $6(8.3)$ & $5(6.3)$ & 0.757 \\
\hline d-TGA, all $(n=165)^{\mathrm{b}}$ & $80(100.0)$ & $85(100.0)$ & \\
NEC, overall & $1(1.3)$ & $1(1.2)$ & 1.000 \\
NEC, after cardiac surgery & $0(0.0)$ & $0(0.0)$ & 1.000 \\
NEC-related mortality & $0(0.0)$ & $1(1.2)$ & 1.000 \\
Mortality, overall & $6(7.5)$ & $4(4.7)$ & 0.526 \\
\hline HLHS $(n=59)$ & $30(100.0)$ & $29(100.0)$ & \\
NEC, overall & $1(3.3)$ & $1(3.4)$ & 1.000 \\
NEC, after cardiac surgery & $1(3.3)$ & $0(0.0)$ & 1.000 \\
NEC-related mortality & $0(0.0)$ & $0(0.0)$ & 1.000 \\
Mortality, overall & $8(26.7)$ & $6(20.7)$ & 0.761 \\
\hline
\end{tabular}

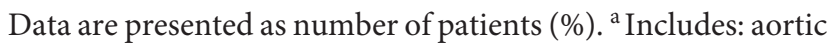
stenosis $+\mathrm{CoA}=4, \mathrm{~d}$-TGA with $\mathrm{CoA}=4$, interrupted aortic $\operatorname{arch}=3$, multiple cardiac anomalies $=1$ (unbalanced atrioventricular septal defect, single right-sided ventricle $+\mathrm{CoA})$ in the no probiotics group, and aortic atresia $+\mathrm{CoA}=1$, aortic atresia + IAA $=1$, aortic stenosis $+\mathrm{CoA}=3$, d-TGA with $\mathrm{CoA}=11$, interrupted aortic arch $=7$ in the probiotics group. ${ }^{\mathrm{b}}$ Including infants with d-TGA and CoA (4 and 11, respectively).

comparison to NEC in premature infants $[2,4,6,11,19]$. In premature infants, NEC is seen as primarily inflammatory disease with secondary necrosis, whereas impaired splanchnic blood flow and oxygen delivery leading to splanchnic ischemia is discussed as major factor in the pathophysiology of NEC in neonates with CHD [11]. Infants with aortic arch obstruction, lesions with significant systemic-to-pulmonary runoff, including aortopulmonary window, hypoplastic left-heart syndrome, and truncus arteriosus, and episodes of low cardiac output are predisposed to mesenteric circulatory insufficiency and have been shown to be at high risk for NEC [2]. Apart from obstruction of systemic circulation or "pulmonary steal" phenomenon, complex CHD can lead to low saturated arterial blood directed towards the lower parts of the body, forming an additional risk factor for (chronic) intestinal hypoxia [11]. Altered mesenteric blood flow in Doppler and lower splanchnic regional oxygenation, as measured by means of near-infrared spectroscopy, have been shown to be associated with development of NEC in infants with critical CHD [21-23].
Although hypoperfusion and hypoxia are postulated to be inciting events in the development of NEC in infants with $\mathrm{CHD}$, the process is nevertheless likely to be multifactorial, and the role of gut microbial colonization in the pathogenesis of NEC in newborns with CHD is still unknown [14]. Acute mesenteric hypoperfusion and chronic hypoxia may alter the development of gut microbiota and the gut barrier integrity, or lead to a chronic state of inflammation $[3,12]$. In older children with cyanotic CHD, chronic hypoxia results in an altered diversity of gut microbial communities with a marked decrease in Lactobacilli [24]. Changes in the gut microbial community structure and function and potential modifications with probiotic bacteria might influence the risk for NEC [12]. Ellis et al. [25] described significant intestinal dysbiosis in CHD patients of at least 34 weeks of gestational age, characterized by decreased total bacteria and decreased Actinobacteria (predominantly Bifidobacteria). However, oral administration of Bifidobacterium infantis did not result in an increase of fecal Bifidobacteria in this pilot study. In contrast, perioperative administration of the probiotic Bifidobacterium breve to neonates undergoing surgery for $\mathrm{CHD}$ significantly changed their intestinal environment [13]. These diverging results raise the question whether or not other influence factors such as enteral feeding, antibiotic treatment, and hypoxia play a more dominant role in shaping the intestinal microbiota, compared to probiotics [25].

Despite different pathophysiological mechanisms of NEC in premature and CHD patients, the use of probiotics for prevention of NEC has been repeatedly discussed, but current evidence is extremely limited $[11,12,14]$. The only published study evaluating the association between probiotic bacteria and NEC, a small prospective, single-center, randomized controlled trial on 100 infants from Turkey, showed a significant reduction of NEC rate $(10 \mathrm{vs.} 0 \%)$ and mortality ( 28 vs. $10 \%$ ) in infants with cyanotic CHD who received a synbiotic preparation containing Bifidobacterium lactis and inulin [26]. No study has yet investigated the effect of probiotic mixtures on NEC in CHD patients.

Our retrospective observational study has notable limitations. The study is not inclusive of all known and suspected risk factors associated with NEC. Due to the long study period of 10 years, clinical management of CHD patients might have changed over time, as indicated by less frequent mechanical ventilation in the intervention group, and whether these changes influence the frequency of NEC and make the comparison of the two study cohorts less conclusive is unclear. Subgroup analyses might not be adequately powered and only be useful to generate hypotheses. Lack of analysis for fecal microbiota impedes us to investigate po- 
tential changes in the composition of the microbiota related to the administration of probiotics. For safety analysis, blood cultures were not available for all infants for investigation of systemic infection from probiotic species. Furthermore, the incidence of probiotic bacteremia might be underestimated due to the practice of requesting only aerobic blood cultures for neonates with suspected infection.

The investigation of a potential impact of probiotics on an already low frequency of NEC in infants with ductal-dependent $\mathrm{CHD}$ is challenging. However, our results warrant further research. A well-designed multicenter trial to further investigate whether the simple approach of administering probiotics is effective to prevent NEC in the high-risk population of newborns with ductal-dependent CHD would be of great interest. Assuming a 50\% reduction of NEC based on a low NEC incidence of $2.4 \%$ as found in our cohort, a clinical trial would need to include almost 4,000 patients with ductal-dependent CHD, an impossible task for a single center. Despite the limitations, this is the first study to investigate the potential impact of probiotic mixtures on NEC in newborns with ductal-dependent cardiac malformations.

\section{Conclusion}

Enteral administration of a dual-strain probiotic containing Bifidobacterium infantis and Lactobacillus acidophilus might have the potential to reduce NEC in infants with ductal-dependent CHD, particularly in those with reduced intestinal perfusion.

\section{Statement of Ethics}

Written informed consent of the study participants (or their parents or guardians) was not obtained, as all data were collected as part of routine clinical care and have been anonymized for the purpose of analysis and presentation. Publication of the results was approved by the Institutional Review Board (Charite Berlin, EA2/069/17).

\section{Conflict of Interest Statement}

The authors have no conflict of interest to declare.

\section{Funding Sources}

The authors have no source of funding to declare.

\section{Author Contributions}

L.K. collected, analyzed, and interpreted the data and drafted the initial manuscript. C.B. contributed to the study design, interpretation of data, and critically reviewed the manuscript for intellectual content. F.B. contributed to the interpretation of data, and critically reviewed the manuscript for intellectual content. V.B. conceptualized and designed the study, contributed in data collection and analysis, supervised data collection and interpretation, and critically reviewed and revised the manuscript for important intellectual content. All authors approved the final manuscript as submitted and agree to be accountable for all aspects of the work.

\section{References}

1 Lin PW, Stoll BJ. Necrotising enterocolitis. Lancet. 2006 Oct;368(9543):1271-83.

2 McElhinney DB, Hedrick HL, Bush DM, Pereira GR, Stafford PW, Gaynor JW, et al. Necrotizing enterocolitis in neonates with congenital heart disease: risk factors and outcomes. Pediatrics. 2000 Nov;106(5):1080-7.

3 Motta C, Scott W, Mahony L, Koch J, Wyckoff $\mathrm{M}$, Reisch J, et al. The association of congenital heart disease with necrotizing enterocolitis in preterm infants: a birth cohort study. J Perinatol. 2015 Nov;35(11):949-53.

4 Lau PE, Cruz SM, Ocampo EC, Nuthakki S, Style CC, Lee TC, et al. Necrotizing enterocolitis in patients with congenital heart disease: a single center experience. J Pediatr Surg. 2018 May;53(5):914-7.

5 Luce WA, Schwartz RM, Beauseau W, Giannone PJ, Boettner BL, Cheatham JP, et al. Necrotizing enterocolitis in neonates undergoing the hybrid approach to complex congenital heart disease. Pediatr Crit Care Med. 2011 Jan;12(1):46-51.

6 Siano E, Lauriti G, Ceccanti S, Zani A. Cardiogenic Necrotizing Enterocolitis: A Clinically Distinct Entity from Classical Necrotizing Enterocolitis. Eur J Pediatr Surg. 2019 Feb; 29(1):14-22.

7 Neu J, Walker WA. Necrotizing enterocolitis. N Engl J Med. 2011 Jan;364(3):255-64.

8 Martin CR, Walker WA. Probiotics: role in pathophysiology and prevention in necrotizing enterocolitis. Semin Perinatol. 2008 Apr; 32(2):127-37.

9 AlFaleh K, Anabrees J. Probiotics for prevention of necrotizing enterocolitis in preterm infants. Cochrane Database Syst Rev. 2014 Apr;(4):CD005496.

10 Dermyshi E, Wang Y, Yan C, Hong W, Qiu G, Gong X, et al. The "Golden Age" of Probiotics:
A Systematic Review and Meta-Analysis of Randomized and Observational Studies in Preterm Infants. Neonatology. 2017;112(1): 9-23.

11 Bubberman JM, van Zoonen A, Bruggink $\mathrm{JL}$, van der Heide M, Berger RM, Bos AF, et al. Necrotizing Enterocolitis Associated with Congenital Heart Disease: A Different Entity? J Pediatr Surg. 2019 Sep;54(9):175560.

12 Ellis CL, Rutledge JC, Underwood MA. Intestinal microbiota and blue baby syndrome: probiotic therapy for term neonates with cyanotic congenital heart disease. Gut Microbes. 2010 Nov-Dec;1(6):359-66.

13 Umenai T, Shime N, Asahara T, Nomoto K, Itoi $\mathrm{T}$. A pilot study of Bifidobacterium breve in neonates undergoing surgery for congenital heart disease. J Intensive Care. 2014 Jun; 2(1):36.
Probiotics for NEC Prevention in Neonates with Congenital Heart Disease
Neonatology 2020;117:569-576 DOI: $10.1159 / 000508831$ 
14 Karpen HE. Nutrition in the Cardiac Newborns: Evidence-Based Nutrition Guidelines for Cardiac Newborns. Clin Perinatol. 2016 Mar;43(1):131-45.

15 Alfaleh K, Bassler D. Probiotics for prevention of necrotizing enterocolitis in preterm infants. Cochrane Database Syst Rev. 2008 Jan 23;(1):CD005496.

16 Guthmann F, Arlettaz Mieth RP, Bucher HU, Bührer C. Short courses of dual-strain probiotics appear to be effective in reducing necrotising enterocolitis. Acta Paediatr. 2016 Mar; 105(3):255-9.

17 Bell MJ, Ternberg JL, Feigin RD, Keating JP, Marshall R, Barton L, et al. Neonatal necrotizing enterocolitis. Therapeutic decisions based upon clinical staging. Ann Surg. 1978 Jan; 187(1):1-7.

18 Penders J, Thijs C, Vink C, Stelma FF, Snijders B, Kummeling I, et al. Factors influencing the composition of the intestinal microbiota in early infancy. Pediatrics. 2006 Aug; 118(2):511-21.
19 Rose AT, Patel RM. A critical analysis of risk factors for necrotizing enterocolitis. Semin Fetal Neonatal Med. 2018 Dec;23(6):374-9.

20 Becker KC, Hornik CP, Cotten CM, Clark RH, Hill KD, Smith PB, et al. Necrotizing enterocolitis in infants with ductal-dependent congenital heart disease. Am J Perinatol. 2015 Jun;32(7):633-8.

21 Carlo WF, Kimball TR, Michelfelder EC, Border WL. Persistent diastolic flow reversal in abdominal aortic Doppler-flow profiles is associated with an increased risk of necrotizing enterocolitis in term infants with congenital heart disease. Pediatrics. 2007 Feb;119(2): $330-5$.

22 DeWitt AG, Charpie JR, Donohue JE, Yu S, Owens GE. Splanchnic near-infrared spectroscopy and risk of necrotizing enterocolitis after neonatal heart surgery. Pediatr Cardiol. 2014 Oct;35(7):1286-94.
23 Stapleton GE, Eble BK, Dickerson HA, Andropoulos DB, Chang AC. Mesenteric oxygen desaturation in an infant with congenital heart disease and necrotizing enterocolitis. Tex Heart Inst J. 2007;34(4):442-4.

24 Xing J, Ying Y, Mao C, Liu Y, Wang T, Zhao $\mathrm{Q}$, et al. Hypoxia induces senescence of bone marrow mesenchymal stem cells via altered gut microbiota. Nat Commun. 2018 May; 9(1):2020.

25 Ellis CL, Bokulich NA, Kalanetra KM, Mirmiran M, Elumalai J, Haapanen L, et al. Probiotic administration in congenital heart disease: a pilot study. J Perinatol. 2013 Sep;33(9): 691-7.

26 Dilli D, Aydin B, Zenciroğlu A, Özyazici E, Beken S, Okumuss N. Treatment outcomes of infants with cyanotic congenital heart disease treated with synbiotics. Pediatrics. 2013 Oct; 132(4):e932-8. 